Recherches en didactique des langues et des cultures

Les cahiers de l'Acedle

14-2 | 2017

Dialogisme et discours en situations didactiques

\title{
Discours enseignant, dialogisme et idéologies académiques
}

Le cas des formulations d'appréciation dans des livrets d'évaluation

$\mathrm{CM} 2-6^{\mathrm{e}}$ d'élèves issus de la communauté gitane

Jérémi Sauvage

\section{OpenEdition}

12 Journals

Édition électronique

URL : http://journals.openedition.org/rdlc/1869

DOI : $10.4000 /$ rdlc. 1869

ISSN : 1958-5772

Éditeur

ACEDLE

Référence électronique

Jérémi Sauvage, "Discours enseignant, dialogisme et idéologies académiques », Recherches en

didactique des langues et des cultures [En ligne], 14-2 I 2017, mis en ligne le 15 juin 2017, consulté le 06 mai 2019. URL : http://journals.openedition.org/rdlc/1869; DOI : 10.4000/rdlc.1869

Ce document a été généré automatiquement le 6 mai 2019.

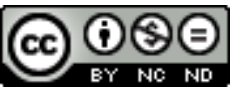

Recherches en didactique des langues et des cultures is licensed under a Creative Commons AttributionNonCommercial-NoDerivatives 4.0 International License 


\section{Discours enseignant, dialogisme et idéologies académiques}

Le cas des formulations d'appréciation dans des livrets d'évaluation $\mathrm{CM} 2-6^{\mathrm{e}}$ d'élèves issus de la communauté gitane

Jérémi Sauvage

\section{Contextualisation, discours et dialogisme}

1 Nous souhaitons dans le présent article nous intéresser à une situation énonciative particulière : la production d'un discours écrit construit par des enseignants de CM2 à destination d'enseignants de collège, dans le cadre d'un document officiel que sont la «fiche de liaison » et le « livret de compétences ». Ce document a pour but, d'une part, d'établir un bilan sur le niveau général de l'élève, d'autre part, d'aider l'équipe pédagogique du collège à organiser la suite de la formation pour favoriser la poursuite des études de l'élève. Cette fiche s'inscrit dans une réflexion générale à propos de la « continuité inter-degré » (Demarcy \& Zarkhartchouk, 2007 ; MEN, 2013). Or, au-delà de la relation duelle strictement professionnelle (entre deux professeurs), cette fiche sera également lue par l'élève et sa famille, ce qui complexifie de manière significative l'énonciation du message. En outre, nous posons l'hypothèse que le discours construit par l'enseignant, s'il est le résultat du sujet-émetteur, est aussi le résultat de représentations sociales participant aux activités cognitives de l'individu (Bronckart, 1997; Sauvage, 2015). Ce principe dialogique interdiscursif apparaît alors dans la situation énonciative à la fois en amont, dans la constitution-même du discours, et en aval, au niveau des destinataires de ce discours.

Nous considérons comme doxa une opinion influant telle ou telle pratique jusqu'à devenir une idéologie (Barthes, 1970). Nos représentations individuelles du monde, si elles sont sémiotisées, sont en rapport avec des représentations sociales, de nature collective, lesquelles participent à l'élaboration de nos discours. Les discours des enseignants sont alors en adéquation avec les normes discursives académiques et les représentations sociales qui leur sont associées. Ce sera par exemple le cas de formulations codées, 
attendues et répandues, dont on peut se demander si elles correspondent vraiment à la situation pédagogique de l'élève auquel elle se réfère ("Très bon travail ", "Elève sérieux », « Des lacunes en grammaire », etc.).

On se demandera alors quelle marge de manœuvre l'enseignant de primaire peut avoir dans la formulation de ses propres appréciations. Tous les enseignants ont un jour été confrontés à la production de LA formulation, celle qui doit rendre compte du travail et du niveau scolaires d'un élève, et donc, de facto, ne ressembler à aucune des autres destinées aux élèves de la classe (ce qui déplace la réflexion sur le terrain de la différenciation pédagogique - Kahn, 2010). Mais si l'on se pose la question du sens de ces formulations, cela nous renvoie immanquablement à leur utilité. A qui sont-elles destinées et avec quel objectif? Peut-on réduire son auteur à l'enseignant-scripteur luimême? Nous proposons dans cet article de nous interroger sur ces points pour montrer qu'au-delà des apparences convenues, il existe un croisement dialogique, à la fois dans la situation d'énonciation, et également au regard des pensées collectives qui sont sousjacentes. Ces réflexions sur l'évaluation en langue sont un domaine foisonnant comme en témoigne le travail de Huver (2014).

En essayant de ne pas répéter les aspects terminologiques et conceptuels déjà développés dans les pages de ce numéro, nous souhaitons préciser dans cette section l'acception dans laquelle nous nous inscrivons lorsque nous employons le terme dialogisme. Les concepts de dialogisme, de polyphonie (Bakhtine-Volochinov, 1929/77) et d'interactionnisme socio-discursif (Bronckart, 1997) peuvent mettre en lumière le rôle déterminant du contexte social et linguistique sur la production linguistique. Les concepts de dialogisme et de polyphonie soulignent le fait que l'énoncé produit est directement lié à l'autre (Bres et al., 2005). De la même manière, l'énoncé anticipe la réponse que le locuteur attend du destinataire. Cette orientation du discours affecte la matérialité de l'énoncé au niveau micro-textuel et dans son expression de temporalité (Todorov, 1981 ; Vion, 1994).

\section{Corpus}

Dans le cadre de cet article, nous avons collecté l'avis d'enseignants à propos du niveau global de trois élèves gitans dans le cadre du projet de recherche Gypsilang piloté par Auger (Auger \& Matheu, 2016) sur les élèves gitans. Dans le cadre de cette étude, nous avions accès aux documents concernant 4 élèves: Indila, Mélissa, Emile et Angelo (les prénoms ont été changés).

\section{Un public spécifique}

6 Peu d'études existent à propos de la scolarisation des enfants gitans en France. Il faut noter tout de même celles de Demeulenaere (2015) ou Decroix et Girodeau (1998). Ces derniers reviennent notamment sur le sens de l'école :

L'école n'a pas la même fonctionnalité pour les Gitans et pour les non-Gitans. Pour le Gitan, tout est orienté autour du groupe, l'important est ce qui peut être utile au groupe, le rôle de chacun au sein de ce dernier, la réussite personnelle n'est pas aussi importante. Les valeurs de la communauté gitane relèvent d'un état antérieur de notre société ; ces dernières sont en contradiction avec les valeurs de l'école républicaine. La modernité qui a fait s'adapter les autres sociétés, n'a pas été intégrée par la communauté gitane, cette dernière au lieu de l'utiliser, ne peut que la subir. (Decroix \& Girodeau, 1998) 
Ainsi, la non-appétence supposée des Gitans pour l'école pourrait trouver sa source dans des représentations sociétales et dans leur rapport social aux savoirs et à l'instruction, sans oublier le rapport social des enseignants et de l'Institution à ces enfants-élèves.

\section{Données et supports}

$7 \quad$ L'un des objectifs de ce projet a consisté à analyser des productions d'écrits par des élèves gitans afin de reconsidérer le rapport que ces derniers ont à l'écrit. En effet, les deux explications que l'on nous donne régulièrement pour justifier l'échec très important de ce public spécifique sont l'absence d'assiduité d'une part, et la difficulté de l'acculturation à l'écrit de ces élèves du fait que la culture gitane serait exclusivement basée sur l'oral. Or, nous avons pu mettre en évidence que les compétences acquises en dernière année d'école élémentaire sont au moins de la même qualité que celles des enfants non-gitans scolarisés dans les mêmes quartiers, voire les mêmes écoles (Sauvage \& Fleuret, sous presse ; Sauvage, Fleuret \& Auger, sous presse). Parmi nos données, nous nous sommes également intéressé aux productions des enseignants, dont certaines ont attiré notre attention du fait de leur diversité et de leur complexité discursive. Sans objectif d'exhaustivité, nous proposons donc ici, de façon exploratoire, de répertorier des appréciations au contact desquelles il est possible de construire un raisonnement interprétatif d'un point de vue dialogique.

\section{Analyses}

8 Nous proposons, dans la présente analyse, de partir de la situation discursive et de nous intéresser aux marqueurs dialogiques utilisés, notamment les pronoms personnels. Précisons dès maintenant que la taille restreinte du corpus, mais pourtant intégrale au vu du peu d'élèves gitans demandant leur entrée au collège, invite à rester prudent quant à l'exhaustivité des critères typologiques mis en évidence, dans la mesure où nous restons à l'échelle d'études de cas.

\section{La fiche de liaison et le livret de compétences}

9 La spécificité de la fiche de liaison est de synthétiser par écrit le niveau général de l'élève, de rendre compte d'un projet de formation et de pointer les compétences à travailler prioritairement ${ }^{1}$. Une commission pédagogique se réunit et un entretien avec l'élève a également lieu afin de déterminer le plus précisément possible où il se situe dans sa scolarité et comment l'aider au mieux à réussir dans la poursuite de sa scolarité. Ce type de document est malgré tout complexe sur le plan dialogique puisque tous les acteurs sont destinataires du message :

- l'enseignant qui formalise son appréciation pour lui-même ;

- ses collègues du collège à qui, sur le plan social, le document est destiné ;

- l'élève à qui l'enseignant répète depuis toujours que «l'appréciation compte plus que la note » et qui se retrouve confronté à sa propre situation scolaire ;

- la famille de l'élève, qu'elle sache lire ou non, que l'on informe officiellement de cette situation et des difficultés et réussites de l'enfant ; 
- l'Institution scolaire enfin, puisque chacun de ces types de document sera précieusement conservé ; il est en effet important de garder une trace écrite dans ce genre de démarche en cas de problème futur.

Le livret personnel de compétences ${ }^{2}$ est par contre un document à vocation pédagogique, informative et constructive. Il suit l'élève tout au long de sa scolarité et permet d'avoir un regard global sur l'évolution de l'acquisition des compétences visées en conformité avec les instructions officielles (Programmes et Socle commun). D'un point de vue discursif, les destinataires pressentis sont alors :

- l'élève, qui est censé prendre conscience de ses lacunes, de ses réussites, de ses progrès ;

- la famille, à qui l'on transmet une sorte de photographie en l'état du niveau des compétences scolaires de l'élève (le fameux bulletin, carnet de notes) ;

- l'enseignant lui-même qui, de période en période ( 5 fois pendant l'année de $\mathrm{CM} 2$ ), se réfère au passé de l'élève pour tracer la dynamique d'évolution des résultats qu'il a obtenus ;

- l'Institution scolaire, dans la mesure où ce livret de compétences suit l'élève de niveau en niveau, de classe en classe, et même d'établissement en établissement.

\section{Le discours pour le collègue d'après}

Dans ce premier type de discours, l'enseignant construit son appréciation pour son (ses) collègue(s) qui aura(ont) en charge l'élève dans la continuité de la scolarité de ce dernier. C'est ce qu'on peut trouver dans :

Après deux ans passés à l'école, Mélissa a beaucoup progressé et a envie de venir à l'école. Mélissa a un niveau de début $\mathrm{CP}$, la lecture est à travailler en priorité.

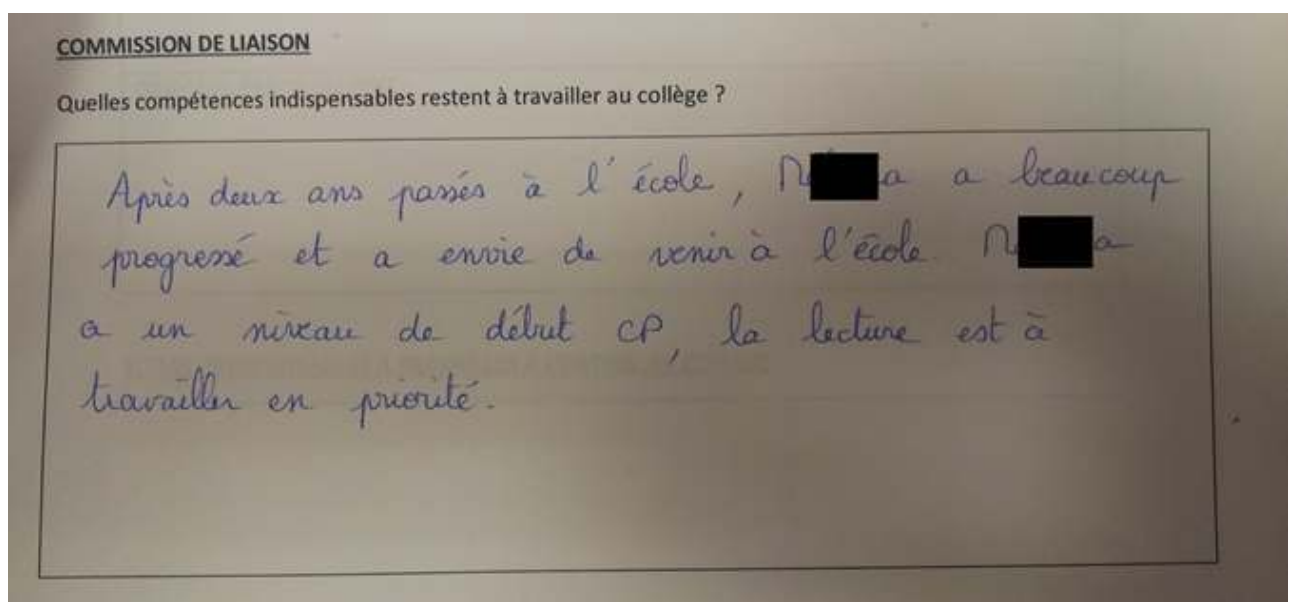

Le contexte énonciatif est le suivant: la commission de liaison doit répondre à la question: Quelles compétences indispensables restent à travailler au collège? Le discours s'adresse donc en priorité à la future équipe pédagogique du nouvel établissement, mais l'élève et sa famille sont également destinataires dans la mesure où ils ont accès au document. L'enseignante de CM2 va donc devoir exprimer le niveau faible de Mélissa tout en essayant de préserver sa face (Goffman, 1974). Après deux ans passés à l'école précise d'emblée aux destinataires enseignants que l'élève n'a été scolarisée qu'à partir de l'âge de 9 ans, ce qui pose le cadre de ses difficultés et devrait susciter l'étonnement. A contrario , du point de vue de Mélissa et sa famille, il ne s'agit que d'un point factuel non-assujetti à interprétations. Mélissa a beaucoup progressé et a envie de venir à l'école donne des éléments 
d'information sur le comportement et l'état d'esprit de l'élève. Ce qui sera compris fort justement comme un compliment par Mélissa et sa famille, aura un impact quelque peu différent pour un enseignant. Elle a beaucoup progressé, soit, mais dans ce contexte particulier où elle est scolarisée depuis seulement deux ans alors que les autres élèves entrant au collège le sont depuis huit années. Toutefois, cette information porte sur la motivation de Mélissa, ce qui la situe par rapport aux autres élèves de sa communauté comme une élève ne posant pas de problème sur ce plan. Cet aspect est très important pour l'équipe pédagogique car la question de la motivation (à quoi bon venir à l'école ?) reste récurrente comme le montre le très fort pourcentage de décrochage scolaire dès la première année de collège ${ }^{3}$. La seconde phrase prend moins de précautions et entre dans le cœur de l'appréciation : Mélissa a un niveau de début de CP signifie que pendant les deux années où elle a été scolarisée à l'école élémentaire, elle a atteint les compétences de fin de maternelle. En d'autres termes, elle ne sait pas lire, ne maitrise pas le geste graphique, ne sait se repérer que jusqu'à 30 sur une bande numérique (sous-entendu des difficultés pour concevoir le temps), etc. Et elle doit entrer au collège. La lecture est à travailler en priorité sous-entend que, puisqu'elle ne sait pas lire, les compétences à viser au collège sont celles du cycle 2 de l'école primaire.

13 L'exemple suivant concerne un autre élève et un autre enseignant. La situation énonciative est la suivante : dans le cas des commissions de liaison, l'enseignant de CM2 doit préciser les priorités sur lesquelles travailler, c'est-à-dire pointer les difficultés les plus importantes pour en faire une priorité dans le projet pédagogique de l'élève. Il faut travailler en maitrise de la langue et la compréhension des textes difficiles. La formulation impersonnelle et lacunaire rend moins évidente l'interprétation du sens pour une personne étrangère au milieu enseignant. Mais l'injonction Il faut renforce le caractère prioritaire du contenu du message.

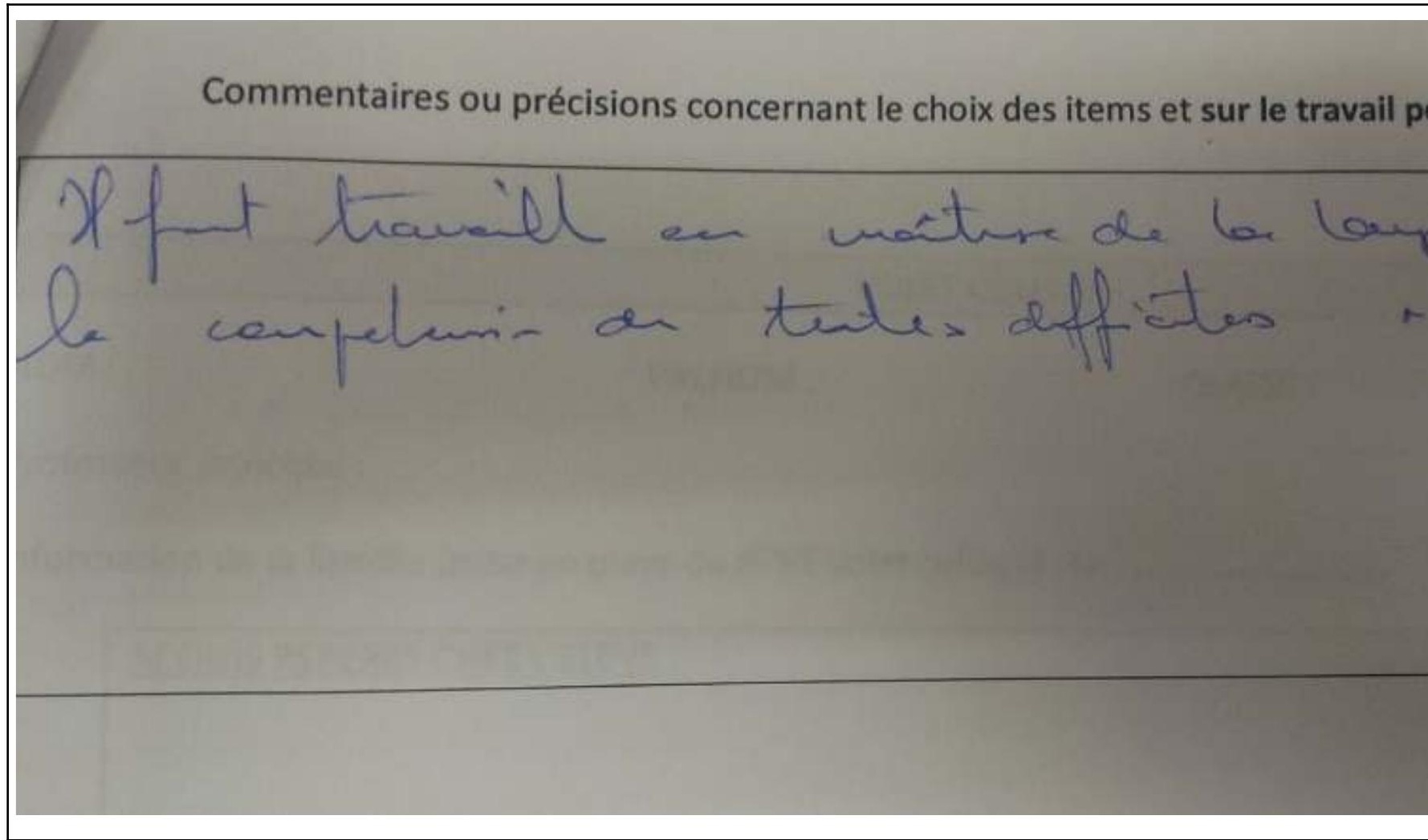


D'un côté, l'enseignant relève les points à améliorer, d'un autre côté le ton injonctif ne laisse pas d'alternative au futur enseignant: travailler la maitrise de la langue et la compréhension de textes difficiles revient à pointer une faiblesse générale de l'élève en langue française. Les termes employés correspondent aux dénominations des instructions officielles et l'absence de conseil pour réussir renforce finalement l'échec de l'enseignant : s'il faut travailler tout cela, c'est que lui a échoué à atteindre ces objectifs. En d'autres termes : «Bon courage cher collègue pour réussir là où j'ai échoué ». Il s'agit donc bien ici d'une utilisation dialogique du discours produit.

\section{Le discours pour l'élève. Le « je » dialogique et la mise en scène de l'enseignant}

15 L'utilisation des pronoms déictiques oblige à être au clair avec la situation énonciative pour savoir qui se cache derrière le 'je'. Dans cet exemple, nous sommes face à un tableau récapitulant les propos d'un entretien entre l'enseignant et l'élève, entretien dans lequel ce dernier doit verbaliser ce qu'il réussit et ce qui reste difficile pour lui. Or la formulation des entrées du tableau interroge : Ce que je réussis bien, Ce qui reste difficile pour moi, Mon défi pour progresser : ce que je dois réussir à faire. Ici, le 'je' est l'élève. Pourtant, ce n'est pas l'élève qui remplit le tableau mais son enseignant. C'est donc ce dernier qui va lui-même écrire : Je lis très bien et je sais travailler en autonomie. Cette année, j'ai réalisé des enregistrements et j'ai été narratrice dans une pièce de théâtre. Derrière ce « je » il y a donc un autre du fait que l'enseignant transcrit des énoncés oraux prononcés par l'élève, ce qui traduit bien une mise en scène discursive (Goffman, 1974). Mais quand un enseignant doit transmettre des informations sur les compétences d'un élève, la priorité concerne ce qui pose problème, à savoir les difficultés que l'élève rencontre toujours malgré ses années d'apprentissage. Et cette fois, dans la colonne de droite, le jeu dialogique des pronoms déictiques se trouve altéré par un autre type de dialogisme : 


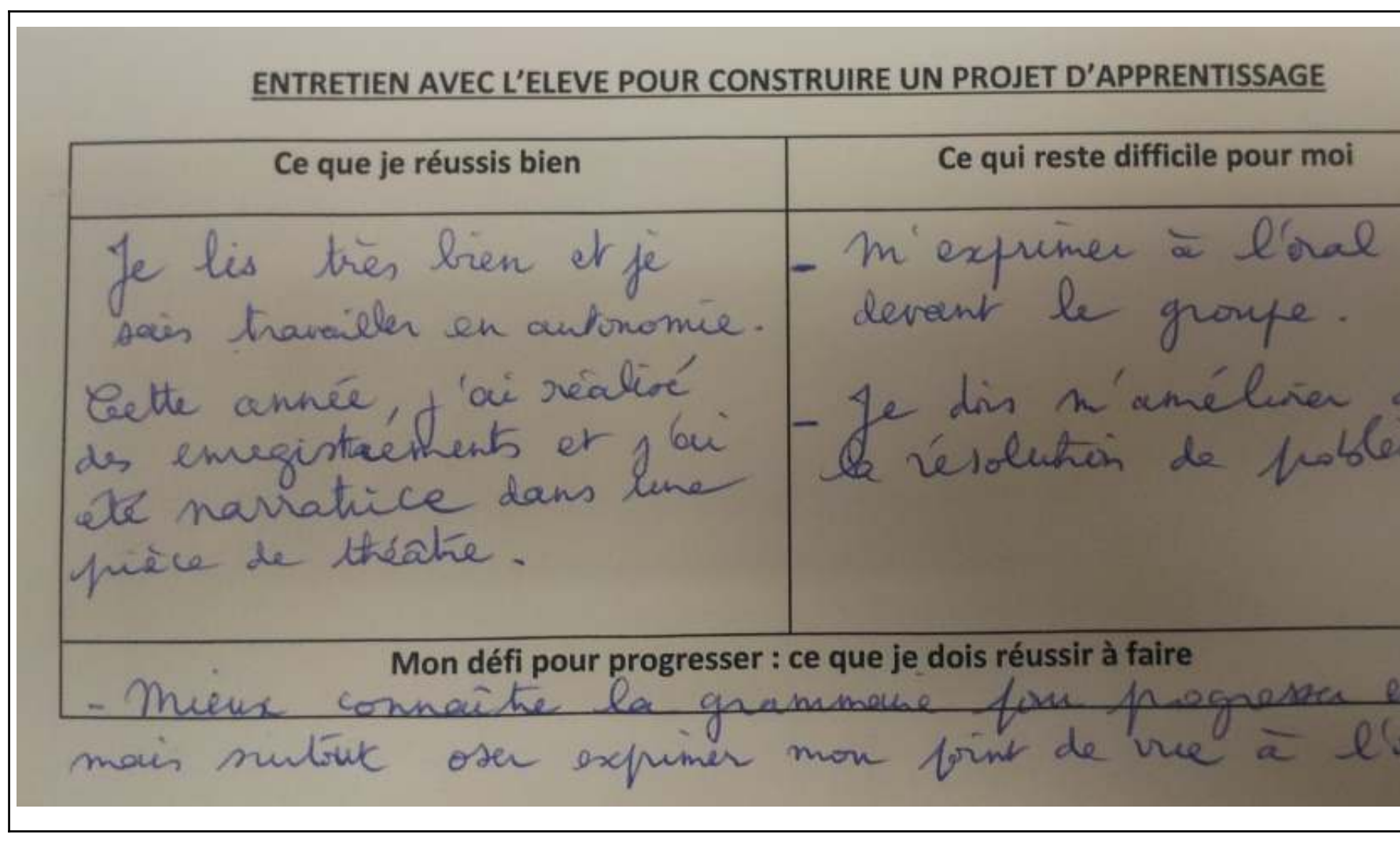

L'enseignante répond ici directement (dialogue ?) avec l'entrée du tableau « Ce qui reste difficile pour moi » ce qui a pour conséquence de faire disparaitre le 'je': M'exprimer à l'oral devant le groupe. L'absence de 'je' (dont on ne garde la trace que par le pronom réfléchi) s'apparente selon nous à une neutralisation énonciative, caractéristique des prescriptions des discours académiques, typique des Instructions officielles du Ministère de l'éducation nationale. En effet, l'une des caractéristiques de ce type de discours est l'utilisation de proposition infinitive : Distinguer les temps du récit et du discours; Dénombrer jusqu'à 100; Se repérer dans le temps et l'espace. Ici, S'exprimer (M'exprimer) à l'oral. On retrouve le même procédé discursif en bas du tableau pour formuler le progrès: Mieux connaitre la grammaire pour progresser en orthographe mais surtout oser exprimer mon point de vue à l'oral. Qui est donc ce 'je' disparu, écrit par un enseignant pour transcrire les paroles d'un élève? Il serait surprenant que l'élève ait ainsi formulé son « défi » (nous n'avons malheureusement pas accès à la situation orale). Nous analysons ici cette formulation de l'enseignant comme une reformulation de la parole de l'élève, reformulation non pas du seul locuteur-enseignant, mais également et surtout, d'une remise en mots en conformité avec les canons du discours académique, avec les idéologies académiques en vigueur.

17 Dans le second exemple, le même enseignant opère, consciemment ou non, la même stratégie formulatoire que précédemment. J'ai une bonne mémoire et je m'exprime bien à l'oral. J'ai compris le fonctionnement de la lecture mais je connais très peu de sons. Je commence à déchiffrer. Ici, au-delà de l'emploi du 'je' comme supra, on retrouve l'utilisation d'un double discours : ce qui est dit ici est que l'élève ne sait pas lire, mais en utilisant une tournure positive. Si je ne connais que très peu de sons et que je commence à déchiffrer, ce qui est catégorisé comme une réussite signifie en fait, par dialogisme, que la conscience phonémique de l'élève n'est pas encore assez structurée pour être sollicitée 
dans le rapport systématique phonème/graphème qui est un préalable au déchiffrage, ce qui est d'ailleurs précisé puisque, si je commence à déchiffrer, je ne sais pas encore déchiffrer.

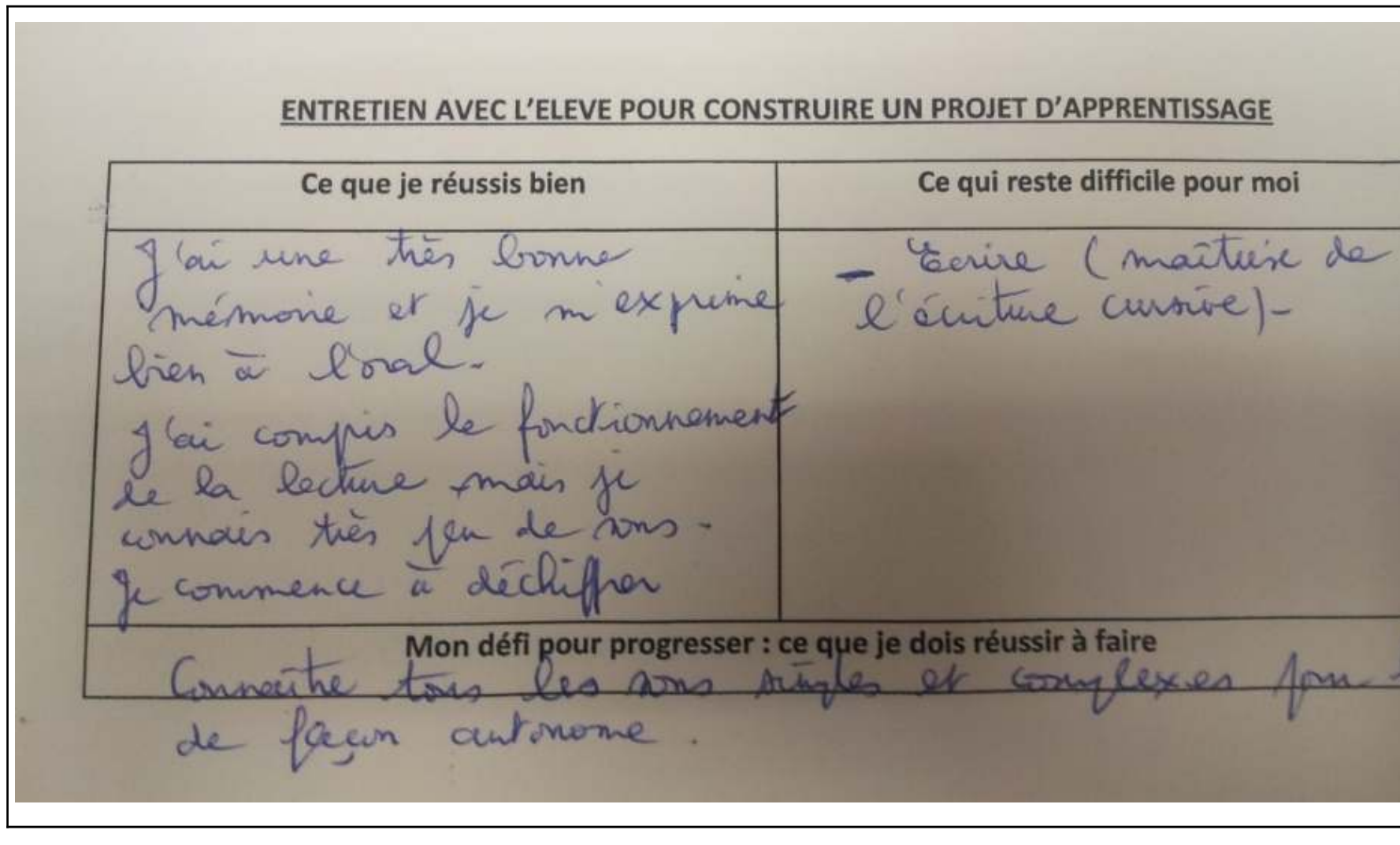

Le corollaire est également évoqué, mais cette fois comme difficulté (colonne de droite) : Ecrire (maitrise de l'écriture cursive). Ici, la précision entre parenthèse sonne comme une reformulation de l'enseignante encore plus explicite que précédemment, qui une fois de plus emprunte des termes académiques : cette difficulté de produire de l'écriture. Il s'agit en fait de formuler «à la manière d'une » compétence tout droit sortie des Instructions officielles: Connaitre tous les sons simples et complexes pour lire de façon autonome. Cette formulation a le mérite de synthétiser les autres commentaires du tableau: l'objectif prioritaire au collège sera d'apprendre à lire et à écrire. En fait, sans recours au dialogisme, les informations de la colonne de gauche (à l'exception de la première phrase) auraient pu se trouver dans la colonne de droite, ce qui aurait comme conséquence, visuellement, (pour l'élève et sa famille) de lister très peu de points positifs et beaucoup de points négatifs. Mais l'équipe pédagogique destinataire de ce discours, elle, ne sera pas dupe et comprendra vite que les difficultés sont bien plus nombreuses que les réussites pour cet élève.

\section{Le discours académique}

19 Le discours académique, dans le sens où nous l'entendons, ne se limite pas aux instructions officielles du Ministère de l'éducation nationale. Comme nous l'avons mentionné au début de cet article, certaines formulations semblent récurrentes d'un enseignant à l'autre, d'une école à l'autre, d'une décennie à l'autre. 


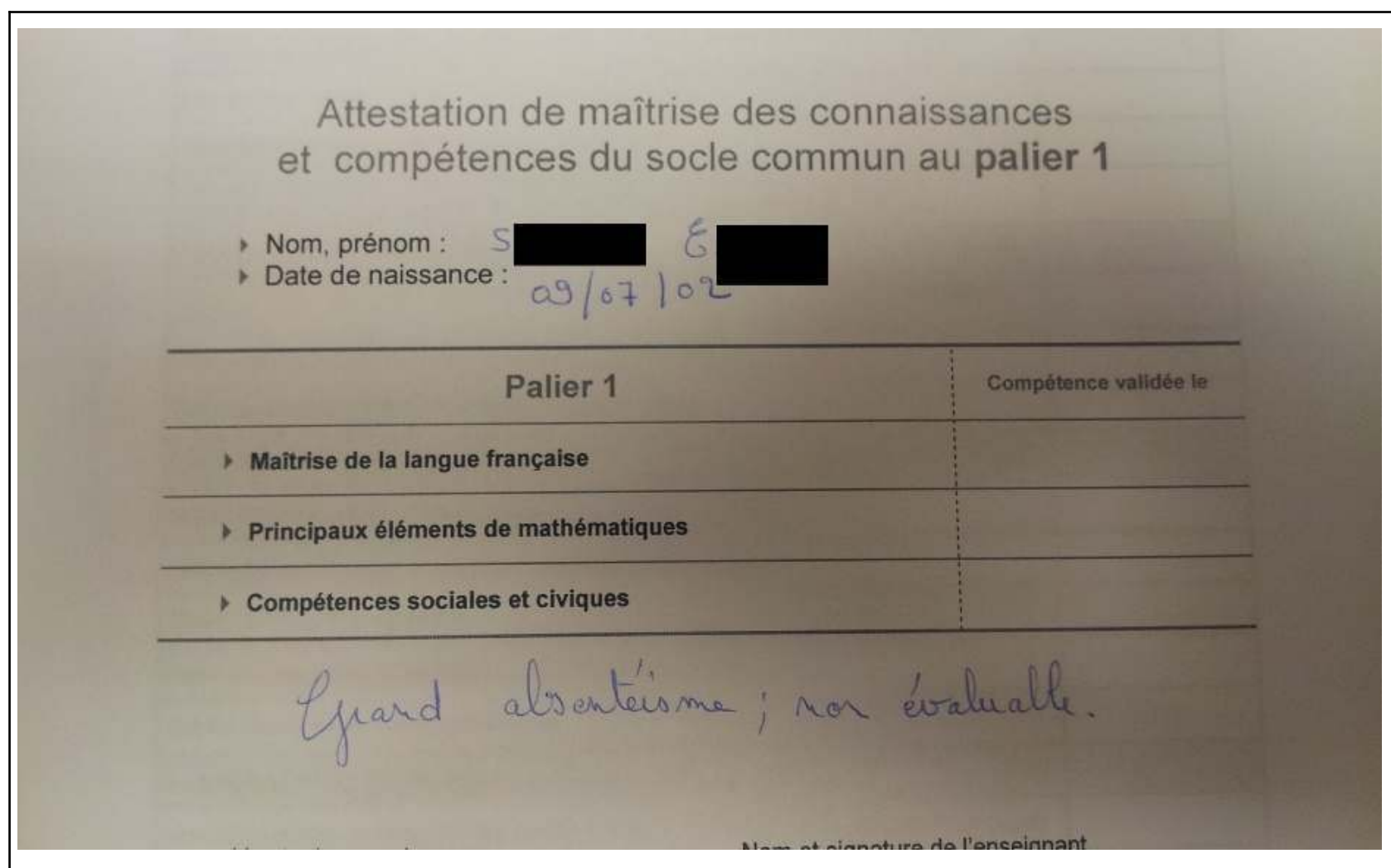

Le fait de ne pas évaluer les compétences du Palier 1 du Socle commun ${ }^{4}$ n'est pas en soi problématique puisqu'on retrouvera régulièrement des appréciations «NE " (nonévaluée) dans bon nombre de livrets de compétences en France. Ce qui nous intéresse ici est le commentaire ajouté alors qu'aucun espace sur la feuille n'invite à rédiger une formulation de ce type: Grand absentéisme, non-évaluable. De la même manière que nous avons constaté que les évaluations nationales de certains élèves pouvaient être écartées des calculs statistiques d'une Inspection académique (pour ne pas tirer les chiffres vers le bas), l'enseignant semble ici dans l'obligation de se justifier. Ce n'est pas que je ne veux pas, mais je ne peux pas évaluer ces compétences chez cet élève. Tout ceci reste finalement assez confortable: je ne dis pas que l'élève a de mauvais résultats. En même temps, s'il ne vient pas en classe, il y a peu de chance que ses compétences soient validées. Mais ce n'est pas de ma faute, moi l'enseignant qui n'aurai pas réussi à atteindre un objectif, puisque Emile est rarement présent. Il aurait été beaucoup plus gênant pour l'enseignant de pointer des lacunes sans absences de l'élève. 


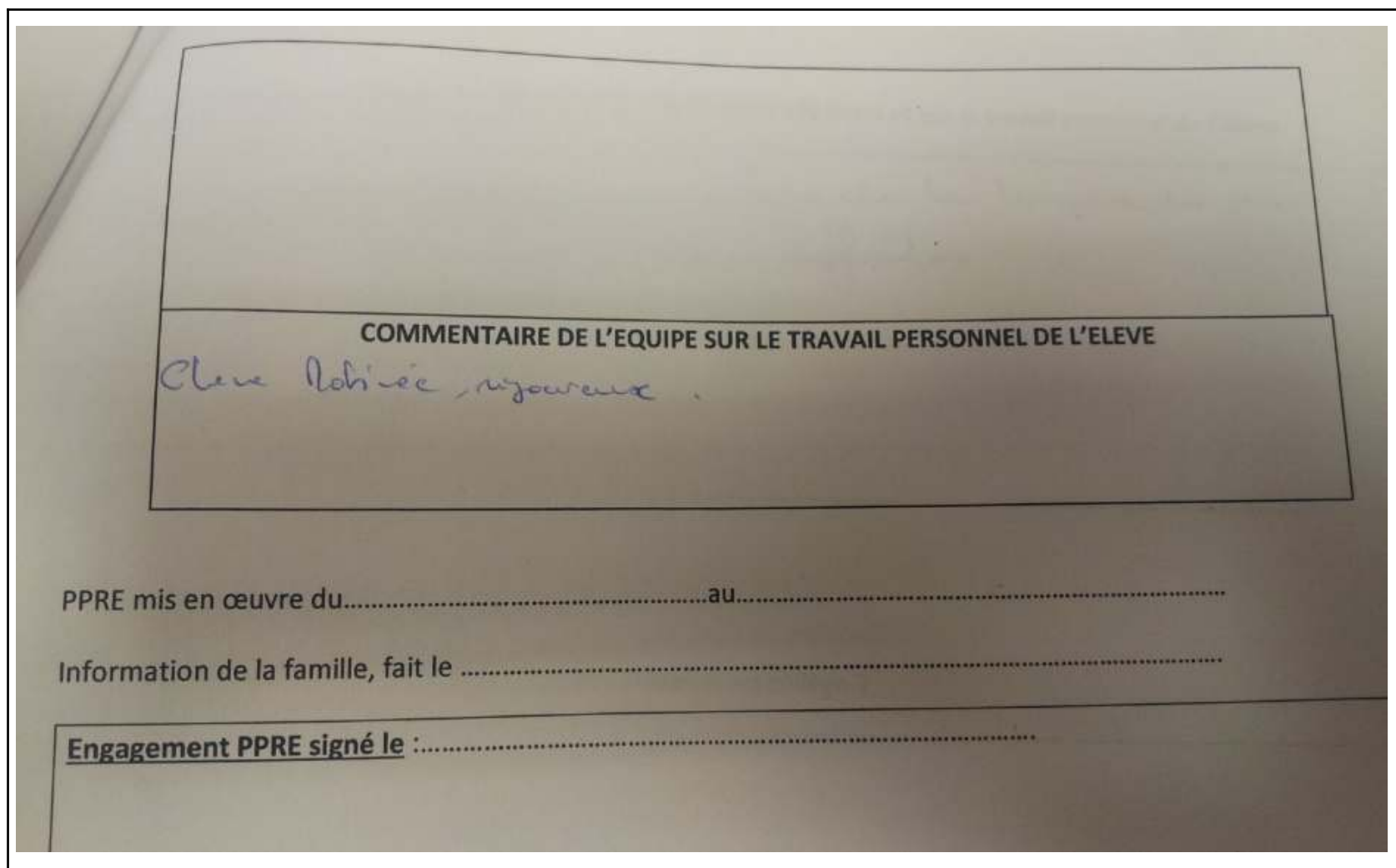

Dans le second exemple, c'est la formulation qui est lacunaire : tout ce qu'on peut dire sur le travail personnel de l'élève est Elève motivée, rigoureuse, ce qui rentre dans les canons académiques du discours enseignant standard, comme nous avons pu le constater comme parent et pratiqué comme enseignant. Or, nous savons que si un élève est motivé, c'est que l'enseignant est motivant (Lieury \& Fenouillet, 2006 ; Plumelle, 2011). Si l'élève n'est pas motivé (sous-entendu par l'enseignant), alors il est absent. Et l'enseignant nonmotivant ne peut pas évaluer ses compétences.

\section{Le sous-entendu négatif}

Finalement, l'objectif communicatif devrait se cantonner au factuel: constater et conseiller l'équipe pédagogique qui prend le relais à partir de ce constat pour mener à bien le projet scolaire de l'élève. Mais les sous-entendus induits par le procédé dialogique peuvent également servir à exprimer un sens qu'une partie des destinataires seulement pourraient comprendre. Curieusement, dans notre corpus, on notera que les «bons élèves » bénéficient d'un discours plus long que les « mauvais » : Angelo a un très bon niveau de langue et une bonne diction. On en déduira, par dialogisme, que ce n'est pas le cas de tous les élèves gitans. Angelo devient donc l'élève rare qui doit attirer toute l'attention de ses futurs enseignants de collège. Entré dans la lecture cette année, il peut décoder les sons simples, c'est-à-dire à 11 ans. On parle ici d'un très bon élève qui ne commence à savoir lire que depuis $1 \mathrm{an}$. Il s'agit donc d'un très bon élève de CM2 avec un niveau CE1 en lecture. 


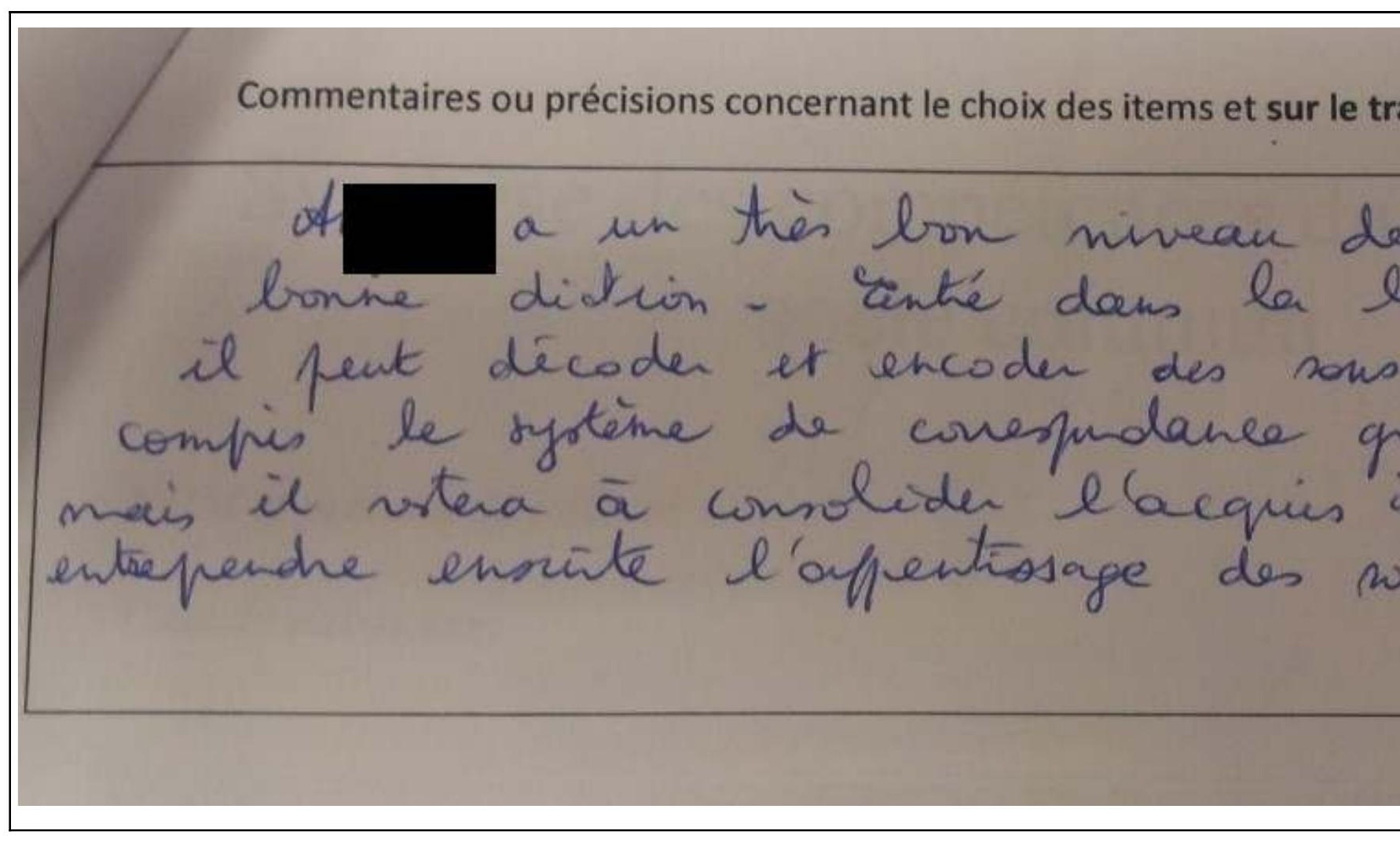

Il a compris le système de correspondance grapho-phonologique, mais il restera à consolider l'acquis à la rentrée pour entreprendre ensuite l'apprentissage des sons complexes. Ce qui veut dire qu'Angelo a un niveau CP très faible et qu'il rentre au collège.

Avec Indila (supra), le sous-entendu atteint son paroxysme.

- Indila doit consolider des compétences en mathématiques (numération : les grands nombres)

- Résolution de problèmes

Et en français : notamment en grammaire relation verbe-sujet / conjugaison pour parfaire son orthographe.

A l'oral, oser s'exprimer.

En fait, selon cette appréciation, et du point de vue d'un enseignant ${ }^{5}$, Indila est à classer dans la catégorie des "mauvaises élèves » en français et en mathématiques, c'est-à-dire en tout. Sous-entendu, elle sera un poids au collège car elle n'a pas le niveau. 


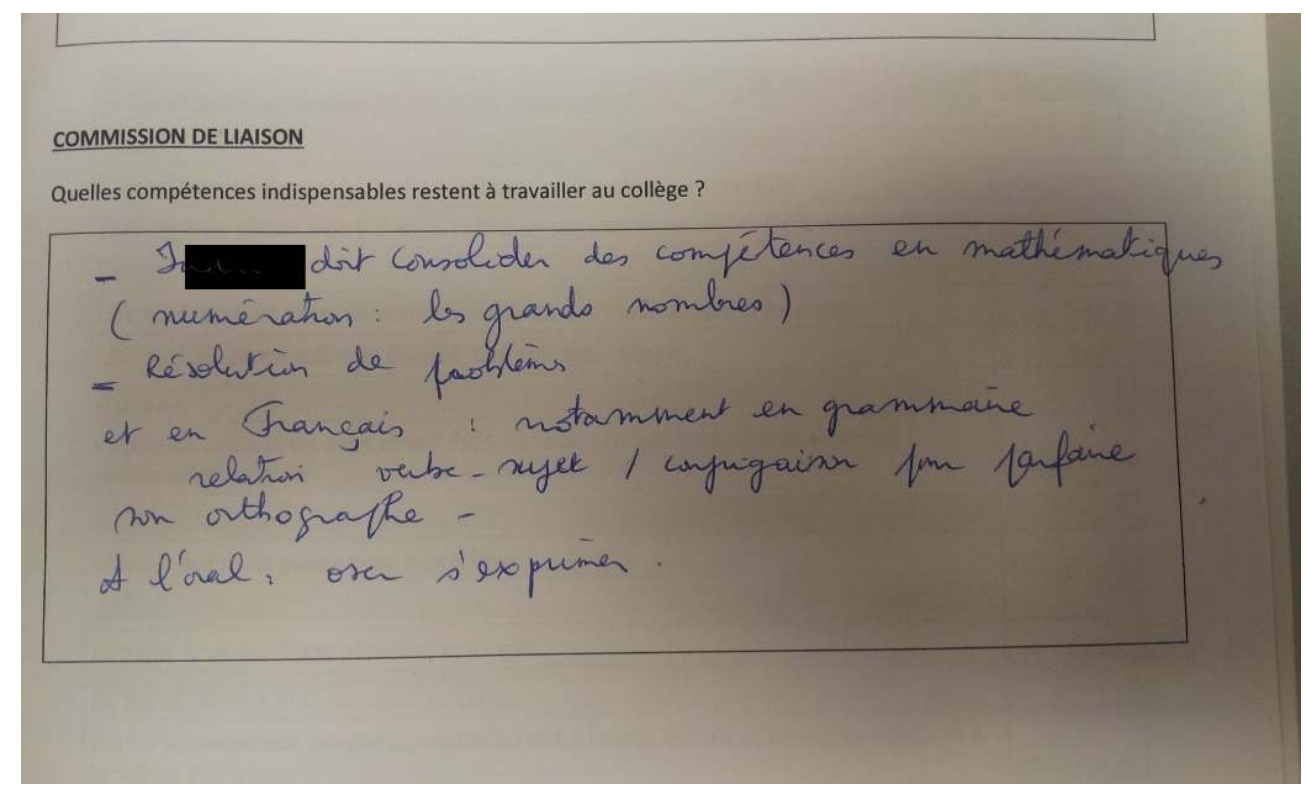

\section{COMMISSION DE LIAISON}

Quelles compétences indispensables restent à travailler au collège ?

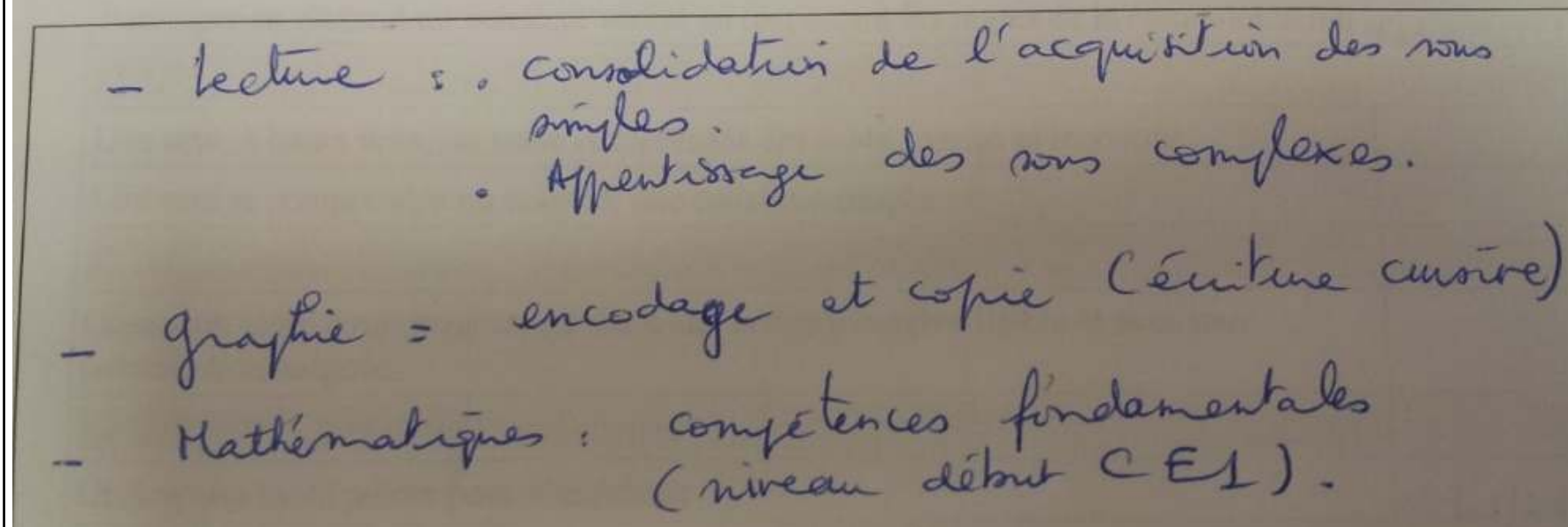

D'ailleurs, le même enseignant pour un autre élève fait également usage du même type de discours, dialogiquement «faussement constructif ».

- Lecture : consolidation de l'acquisition des sons simples

. Apprentissage des sons complexes.

- Graphie : encodage et copie (écriture cursive).

- Mathématiques : compétences fondamentales (niveau début CE1) 
Notre expérience d'enseignant du premier degré nous invite à décoder ce discours. Une fois de plus l'enseignant explique étrangement que Indila est très loin du niveau attendu à l'entrée du collège, qu'elle ne sait ni vraiment lire, ni vraiment écrire, ni vraiment compter. Ainsi, le message réel envoyé par l'enseignant à ses collègues est en fait bien plus violent verbalement (Auger \& Romain, 2015 ; Fracchiolla, Moïse, Romain \& Auger 2013) qu'il n'en a l'air. Même si l'intention est de rester factuel, le procédé dialogique auquel il est difficile d'échapper, donne au sens du message une dimension qui n'est pas clairement exprimée.

\section{Discussion : la formulation constructive vs toute faite}

Nous avons essayé de mettre en évidence des éléments de complexité pour montrer que le discours produit par l'enseignant est parfois grandement sous l'influence d'autres discours (dialogisme interdiscursif) ou de représentations collectives intégrées. La complexité de la situation énonciative est ici intéressante dans la mesure où, en amont, le discours de l'enseignant est sous l'influence d'autres discours de nature académique, mais aussi parce qu'il s'adresse à différents destinataires avec des niveaux de compréhension différents (famille, collègues...). Le discours sous influence (idéologique) académique ne pourra être interprété que par ceux qui sont initiés à son codage. La famille ne l'étant bien évidemment pas dans cette étude, seuls les collègues du collège de l'enseignant seront à même de saisir tout le sens de ces énoncés. En même, temps, on peut également se demander comment il pourrait en être autrement. Car s'ils sont enseignants, les locuteurs étudiés n'en sont pas moins soumis aux règles du discours comme tout locuteur. Il est alors possible de mesurer le poids des discours académiques et leur influence sur les discours produits par les enseignants. Il serait dès lors bien inutile de leur en vouloir. Au contraire, il est nécessaire de porter une attention toute particulière à ces appréciations si chargées en sous-entendus s'opérant par dialogisme.

\section{BIBLIOGRAPHIE}

Auger, N. \& Matheu, N. (2016). « Du gitan au français : d'une langue hors-norme à une langue normée ?». Signes, Discours et Sociétés, n¹6. Discours hors-normes, constructions sociales. http:// www.revue-signes.info/document.php?id=4721.

Auger, N. \& Romain, Ch. (2015). Violence verbale et école. Paris : L'Harmattan.

Auger, N. \& Sauvage, J. (2007). « Des familles d'activités pour favoriser les articulations entre les langues et les variations présentes ou importées dans la classe ». Deyrich, M.-C. \& Kervran, M. (dir.). APLV. Les Langues Modernes. https://hal.archives-ouvertes.fr/hal-01139621.

Bakhtine-Volochinov, M. (1929-77). Le marxisme et la philosophie du langage. Paris : Minuit.

Barthes, R. (1970). S/Z. Paris : Seuil. 
Bres, J., Haillet, P.P., Mellet, S., NElk, H. \& Rosier L. (éds.) (2005). Dialogisme et polyphonie. Approches linguistiques. Bruxelles : De Boeck Duculot.

Bronckart, J.-P. (1997). Activité langagière, textes et discours. Pour un interactionnisme socio-discursif. Neuchâtel et Paris : Delachaux et Niestlé.

Decroix, C. \& Giraudeau, H. (1998). « Scolariser des enfants gitans : une interrogation pour le système ». Ville, Ecole, Intégration, $n^{\circ} 115$. http://www2.cndp.fr/revueVEI/decroix115.htm.

Demarcy, D. \& Zarkhartchouk, J.-M. (dir). (2007). Réussir le passage de l'école au collège. Amiens : CRDP.

Demeulenaere, M. (2015). Le tableau noir des enfants gitans. http://mariedemeulenaere.wix.com/ journalisme\#!le-tableau-noir-des-enfants-gitans/c58q.

Fracchiolla, B., Moïse, Cl., Romain, Ch. \& Auger, N. (dir.) (2013). Violence verbale. Analyses, enjeux et perspectives. Paris : L'Harmattan.

Goffman, E. (1974). Les rites d'interaction. Paris : Minuit.

Huver, E. (2014). De la subjectivité en évaluation à une didactique des langues diversitaire. Mémoire d'Habilitation à diriger les recherches, soutenue à Tours.

Kahn, S. (2010). Pédagogie différenciée et inégalités scolaires. Bruxelles : De Boeck.

Lieury, A. \& Fenouillet, F. (2006). Motivation et réussite scolaire. Paris : Dunod.

MEN, (2013). « Liaison inter-établissement : assurer la continuité des apprentissages ».

Futuroscope, Esen, novembre 2013. http://www.esen.education.fr/fr/ressources-par-type/outilspour-agir/le-film-annuel-des-personnels-de-direction/detail-d-une-fiche/?

$\mathrm{a}=44 \& \mathrm{cHash}=374 \mathrm{e} 54 \mathrm{f} 9 \mathrm{f} 2$.

MEN, (2013). Rapport n 2013-072 Juillet 2013. « La notation et l'évaluation des élèves éclairées par des comparaisons internationales ", à Monsieur le ministre de l'Éducation nationale par Charbonnier, D., Houchot, A., Kerrero, C., Moutoussamy, I., Thollon, F. IGEN.

Ortolang, gypsylang (2015-2016). Corpus « Paroles de locuteurs gitans et non gitans sur trois générations à Perpignan ». https://www.ortolang.fr/market/corpora/prax000931.

Plumelle, B. (2011). Références bibliographiques du dossier « Le plaisir et l'ennui à l'école ». Revue Internationale d'Education de Sèvres, ${ }^{\circ}{ }^{5}$ 57. pp. 155-165.

Sauvage, J. (2015). L'acquisition du langage. Un système complexe. Louvain la Neuve : Academia.

Sauvage, J. \& Fleuret, C. (sous presse). « Le rapport à l'écrit d'élèves gitans et non gitans : analyse comparée de deux dictées dans le cadre des évaluations nationales de CE1 ». In Auger, N. (dir). Enfants gitans en famille et à l'école.

Sauvage, J., Fleuret, C. \& Auger, N. (sous presse). « Etudes des erreurs orthographiques en français langue seconde d'élèves issus de la communauté gitane de perpignan. Une étude de cas ». In Nouveaux Cahiers de la Recherche en Education. Université de Sherbrooke.

Todorov, T. (1981). Mikhail Bakhtine. Le principe dialogique. Paris : Seuil.

Vion, R. (1994). « La linguistique et le dialogue ». Actes du colloque international Dialogique. Université du Maine. Berne : Peter Lang. 


\section{NOTES}

1. http://www.carnet-liaison-prems.com/.

2. http://media.education.gouv.fr/file/27/02/7/livret_personnel_competences_149027.pdf.

3. On nous a relaté que sur les dix dernières années, un seul élève était entré au lycée sur toute la communauté.

4. http://eduscol.education.fr/pid23410/le-socle-commun.html.

5. Je précise que j’ai été moi-même enseignant en école primaire plusieurs années.

\section{RÉSUMÉS}

Nous proposons dans cet article de réfléchir sur le rôle des doxas et des pensées issues d'opinions idéologiques dans le jeu dialogique nécessaire de l'exercice de l'appréciation de l'enseignant du premier degré à destination d'enseignants du collège. Pour ce faire, nous nous appuierons sur un corpus d'appréciations à partir desquelles nous proposerons les prémices d'une typologie dont l'objectif principal sera de mettre en évidence que le « je » de ces discours n'est pas toujours celui que l'on croit.

We propose in this paper to reflect on the part of doxas and thoughts stemming from ideological opinions in the necessary dialogic play of the exercise of the appreciation of the first-level teacher for teachers of the high school. To do this, we will rely on a corpus of appreciations from which we propose the beginnings of a typology whose main objective will be to show that the teacher says "I" it does not always refer to himself.

\section{INDEX}

Mots-clés : dialogisme, interdiscursif, discours enseignant, élèves gitans, didactique

Keywords : dialogism, interdiscursive, teacher's discourse, gypsy students, education

\section{AUTEUR}

\section{JÉRÉMI SAUVAGE}

Université Paul-Valéry, Montpellier 3 - Praxiling, UMR 5267

Jérémi Sauvage est maître de conférences HDR en linguistique et didactique du FLE, spécialiste de l'enseignement et de l'apprentissage de l'oral, en particulier en phonétique et phonologie.

jeremi.sauvage [at] univ-montp3.fr 